\title{
Confocal unstable-resonator semiconductor laser
}

\author{
J. Salzman \\ Bell Communications Research Laboratory, 600 Mountain Avenue, Murray Hill, New Jersey 07974 \\ R. Lang \\ California Institute of Technology, Pasadena, California 91125
}

\section{A. Larson}

Department of Electrical Measurements, Chalmers University of Technology, S-41296 Göteborg, Sweden

A. Yariv

California Institute of Technology, Pasadena, California 91125

Received January 21, 1986; accepted May 20, 1986

\begin{abstract}
GaAs/GaAlAs heterostructure lasers with a monolithic confocal unstable resonator were demonstrated. The curved mirrors satisfying the confocal condition were fabricated by etching. Close to threshold, the lasers operate in a single lateral mode with a nearly collimated output beam. A single-lobe far-field intensity distribution as narrow as $1.9^{\circ}$ full width at half maximum was measured.
\end{abstract}

Laser cavities of the unstable resonator (UR) type have been shown to exhibit many advantages when they are combined with a lasing medium characterized by a high-single-pass gain. ${ }^{1-4}$ The most prominent of these advantages is the potential for increasing the mode volume of a laser oscillator while maintaining high discrimination between transverse modes. As a result, the cavity is relatively insensitive to inhomogeneities in the lasing medium. These properties make UR geometries attractive for semiconductor lasers, ${ }^{5}$ since they may provide an optimal solution to the task of increasing the lateral dimension of the laser and avoiding multilateral mode operation and filamentation.

UR semiconductor lasers were demonstrated previously. ${ }^{6-10}$ However, in all previous work the fabricated UR semiconductor lasers emitted a diverging optical beam. This is a disadvantage in applications in which the coupling of the laser output to optical devices with low numerical aperture is considered. The purpose of this Letter is twofold: (1) to demonstrate the operation of a semiconductor laser with a monolithic confocal UR that emits a nearly collimated output beam ${ }^{11}$ and (2) to show that the fabrication of semiconductor lasers with etched curved mirrors permits the design of lasers with integrated cavities that fit specific optical output requirements.

The confocal UR of positive branch is shown in Fig. 1. The cavity is composed of a large concave mirror $\mathrm{M}_{1}$ and a small convex mirror $\mathrm{M}_{2}$ with radii of curvature $R_{1}$ and $R_{2}$, respectively, satisfying the confocality condition $R_{1}+R_{2}=2 L$, with $L$ the cavity length. The geometrical magnification $m$ is given by the ratio of the mirror lateral sizes $a_{1} / a_{2}$. The output beam is that portion of radiation reflected by $\mathrm{M}_{1}$ that misses strik- ing $\mathrm{M}_{2}$ (Fig. 1). A remarkable feature of this cavity is that its fundamental mode is a collimated two-section beam (or a collimated annular beam in the three-dimensional case).

Figure 2 shows the geometry of the confocal UR semiconductor laser. GaAs/GaAlAs double heterostructures were grown by molecular-beam epitaxy, and conventional photolithographic methods were used to define the desired pattern for mirror etching and metallization. Apart from the slight complication of the need to provide three separate electrical contacts, the fabrication is similar to that of previously reported UR semiconductor lasers. ${ }^{7-10}$ The cavity dimensions are $2 a_{1}=150 \mu \mathrm{m}, 2 a_{2}=30 \mu \mathrm{m}, R_{1}=400 \mu \mathrm{m}$, $R_{2}=-80 \mu \mathrm{m}$, and $L=160 \mu \mathrm{m}$, leading to a geometrical magnification of $m=5$, and an equivalent Fresnel number $N_{\mathrm{eq}}=350$. We note that, unlike in the case of nonsemiconductor UR lasers, in which the cavity is defined by external optics, the output beam here is refracted by a semiconductor-air interface. Reflections from this interface may complicate the mode pattern and reduce the lateral mode discrimination.

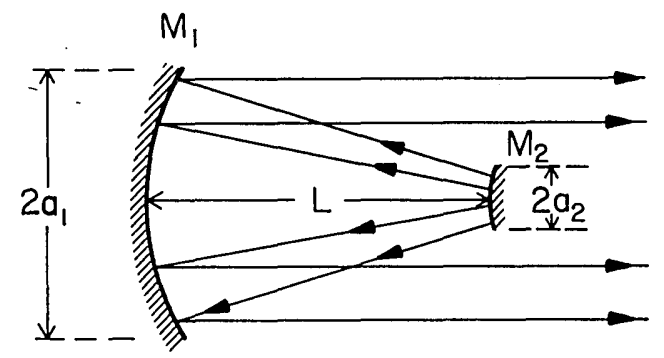

Fig. 1. Geometry of the unstable resonator of positive branch. 


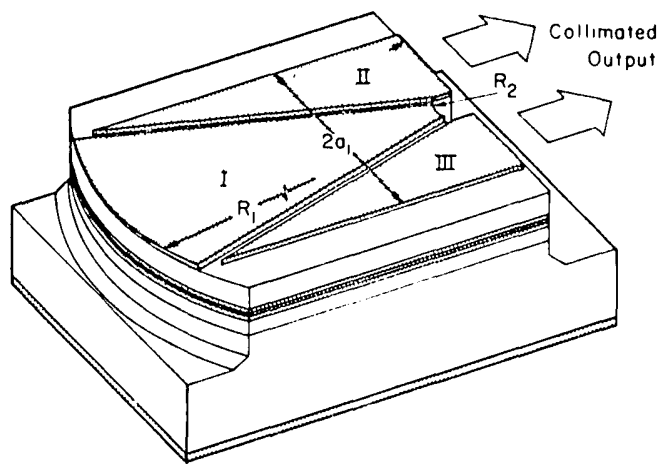

(a)

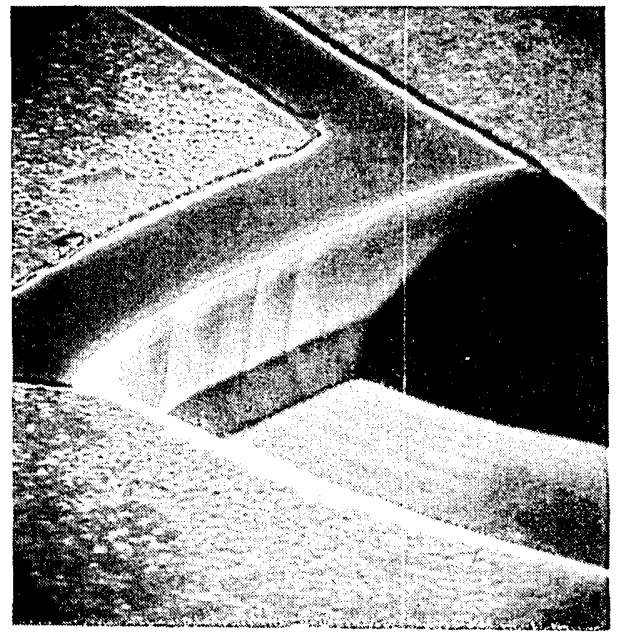

$\stackrel{H}{1 \mu \mathrm{m}}$

(b)

Fig. 2. (a) Schematic drawing of the confocal UR semiconductor laser. Section I is the resonator. Sections II and III are the output couplers. (b) SEM picture of the etched mirror $\mathrm{M}_{2}$.

An additional problem is that the radiation reflected by $M_{1}$ is nearly collimated. Thus the outer parts of the cavity [sections II and III in Fig. 2(a)] are susceptible to regenerative self-focusing and the formation of isolated filaments. These problems can be avoided to some extent by providing separate electrical contacts to the output couplers (sections II and III) so that the central part of the laser - the resonator - can be operated above threshold and the output couplers given a low injection current (pumped to transparency). Furthermore, additional properties of the resonator can be studied by independently changing the current in the different sections.

A scanning electron microscope (SEM) picture of the etched mirror $\mathrm{M}_{2}$ is shown in Fig. 2(b). (The rough sections on top of the device are the $\mathrm{Cr}-\mathrm{Au}$ contact pads.) Here, ripples in the etched mirror of the order of (or less than) $\sim 0.1 \mu \mathrm{m}$ can be observed. Slight misalignment of the mirrors caused by undercut in the etched facets was also noticed in the measured devices.

A rough estimate of the output-beam characteristics was obtained by considering the fundamental lateral mode of a two-dimensional confocal UR in the geometrical approximation. ${ }^{2}$ The near field of this mode consists of a uniformly illuminated, constant-phase aperture of width $2 a_{1}$ with a central obscuration $2 a_{2}$ wide. The far-field intensity distribution $I(\theta)$ corresponding to this aperture is given by

$$
I(\theta)=I_{0} \frac{\sin ^{2}\left[\theta\left(u_{1}-u_{2}\right)\right]}{\left[\theta\left(u_{1}-u_{2}\right)\right]^{2}} \cos ^{2}\left[\theta\left(u_{1}+u_{2}\right)\right],
$$

where $u_{1,2}=a_{1,2} / \lambda, \lambda$ is the optical wavelength, $I_{0}$ is a constant, and $\theta$ is the far-field angle. By inserting the values of $a_{1}$ and $a_{2}$ we obtain the main lobe width of the ideal-cavity far-field pattern $\Delta \theta_{0}=0.27^{\circ}$.

A more rigorous analysis of our device would result in a much more complicated radiation pattern than that predicted by Eq. (1). Diffraction effects at the edges of the mirrors will introduce ripples in the nearfield pattern. ${ }^{12}$ Reflections at the output plane may result in oscillation of high-transverse-order HermiteGaussian modes. ${ }^{13}$ Finally, imperfections in the mirrors, a misalignment of the cavity, and index inhomogeneities may cause distortions and a degradation in the beam quality.

The lasers were tested under pulsed condition, with $200-\mathrm{nsec}$ pulses and a $1-\mathrm{kHz}$ repetition rate, and the output pattern monitored with a vidicon camera. In Fig. 3, the near-field intensity distribution is recorded for different values of the injection current. Figure 3(a) shows the spontaneous emission in sections II and III. When section I is operated below threshold, the

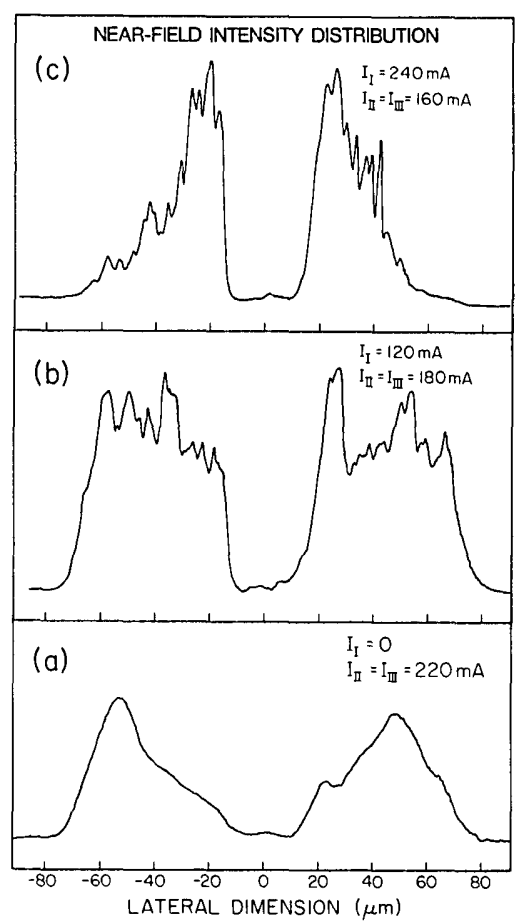

Fig. 3. Near-field intensity distribution of the confocal UR semiconductor laser for different values of the injection current. (a) Spontaneous emission in sections II and III. (b) Section I operated below threshold. (c) Section I operated above threshold. 


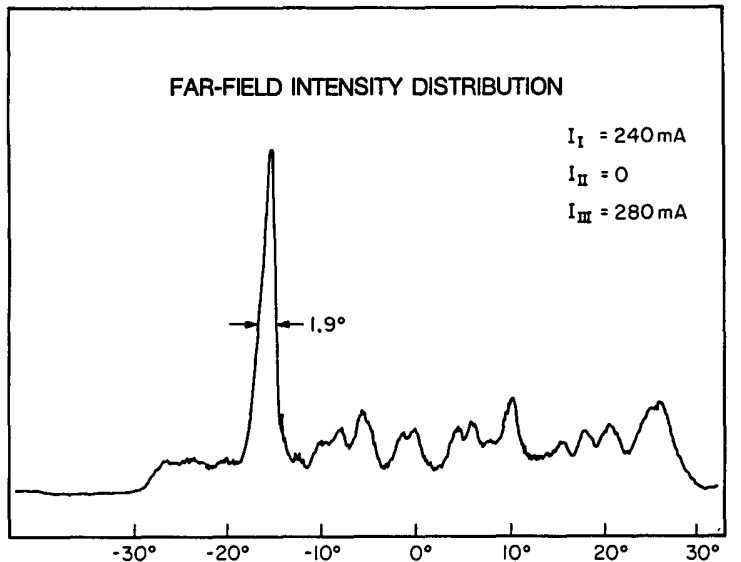

Fig. 4. Far-field intensity distribution with section I above threshold and only one of the output couplers operated.

near-field pattern became nearly constant over the aperture, high-spatial-frequency ripples, characteristic of the confocal UR fundamental mode ${ }^{12}$ appear [Fig. 3(b)], and the two output beams become partially phase locked. When section I (the resonator) is above threshold, the output is peaked in the regions adjacent to the central obscuration, indicating that the cavity is not exactly in the confocal condition [Fig. 3(c)]. However, by measuring the spectrally resolved near field we confirmed that the two beams of Fig. 3(c) were phase locked. ${ }^{14}$ A misalignment of the cavity was revealed also by observing the far-field pattern and changing the injection current in sections II and III. When only one of the sections was operated, the near field resembled half of Fig. 3(c), but a very narrow farfield beam was observed. The position of this beam varied from device to device, with typical off-axis values of $10 \pm 6^{\circ}$. When both sections were operated simultaneously, a complicated interference pattern was observed. In Fig. 4 a far-field intensity distribution with a central peak of FWHM $1.9^{\circ}$, located $16^{\circ}$ off axis, is shown. At higher injection levels, output powers of $\sim 100 \mathrm{~mW}$ were measured within $2.5^{\circ}$ FWHM.

The misalignment problems mentioned above can be understood by considering the sensitivity of the output beam to variations in the confocal cavity parameters. A perturbation analysis of the outputbeam characteristics ${ }^{2}$ results in the following tolerances on $L, R_{1}$, and $R_{2}$ :

$$
\left|\frac{\Delta L}{L}\right| \sim\left|\frac{\Delta R_{1}}{2 L}\right| \sim\left|\frac{\Delta R_{2}}{2 L}\right| \gtrsim \frac{L \lambda}{16 a_{1}^{2}} .
$$

This represents an accuracy of better than $\pm 0.1 \mu \mathrm{m}$ in $L$ and mirror shapes smooth to within $\pm 20 \mathrm{~nm}$. These figures are beyond the resolution of the conventional photolithography used to define the cavity in this Letter.

We would like to mention that no mirror coating was performed on these lasers. By antireflection coating of the output coupling plane, high-reflecting coating of $M_{1}$ and $M_{2}$, and use of a off-axis confocal UR with the same techniques used in the present work, a highpower-narrow-beam semiconductor source can be fabricated. The results reported in this Letter suggest that, with the improvement of submicrometer pattern-transfer techniques (e.g., electron-beam photolithography) and the further development of reliable etching procedures, new cavity configurations can be incorporated monolithically into semiconductor lasers. The resonator geometry can then be designed to fit specific optical output requirements.

This research is supported by grants from the U.S. Army Research Office, the U.S. Air Force Office of Scientific Research, and the U.S. Office of Naval Research and by contract NAS7-918 with the National Aeronautics and Space Administration. J. Salzman would like to acknowledge the support of the Bantrell Postdoctoral Fellowship and the Fullbright Fellowship. R. Lang would like to acknowledge the support of the National Science Foundation.

\section{References}

1. A. E. Siegman, Proc. IEEE 53, 277 (1965).

2. W. F. Krupke and W. R. Sooy, IEEE J. Quantum Electron QE-5, 575 (1969).

3. J. P. Reilly, IEEE J. Quantum Electron. QE-8, 136 (1972).

4. R. A. Chodzko, H. Mirels, F. S. Roehrs, and R. Pedersen, IEEE J. Quantum Electron. QE-9, 523 (1973).

5. A. P. Bogatov, P. G. Eliseev, M. A. Man'ko, G. T. Mikaelyan, and Y. M. Popov, Sov. J. Quantum Electron. 10, 620 (1980).

6. R. R. Craig, L. W. Casperson, O. M. Stafsudd, J. J. J. Yang, G. A. Evans, and R. A. Davidheiser, Electron. Lett. 21, 62 (1985).

7. J. Salzman, T. Venkatesan, R. Lang, M. Mittelstein, and A. Yariv, Appl. Phys. Lett. 46, 218 (1985).

8. M. Mittelstein, J. Salzman, T. Venkatesan, R. Lang, and A. Yariv, Appl. Phys. Lett. 46, 923 (1985).

9. J. Salzman, R. Lang, S. Margalit, and A. Yariv, Appl. Phys. Lett. 47, 9 (1985).

10. J. Salzman, R. Lang, T. Venkatesan, M. Mittelstein, and A. Yariv, Appl. Phys. Lett. 47, 445 (1985).

11. J. Salzman, R. Lang, M. Mittelstein, and A. Yariv, in Digest of Conference on Lasers and Electro-Optics (Optical Society of America, Washington, D.C., 1985), p. 40.

12. D. B. Rensch and A. N. Chester, Appl. Opt. 12, 997 (1973).

13. A. H. Paxton and J. H. Erkkila, Opt. Lett. 1, 166 (1977).

14. The spectrally resolved near field shows several longitudinal modes (as many as 10 modes at high injection levels). The spectra of the two beams in Fig. 3(c) are identical, indicating that they are phase locked. 